\title{
Hypothetical Research Note: Artificial Dark Photosynthesis Models for Capturing Carbon Dioxide into Fuels
}

\author{
Tomonori Kawano ${ }^{1,2,3 *}$, Diego Comparini ${ }^{1,2}$, Omi Uchimura ${ }^{4,5}$ and Kazuya Uezu ${ }^{1}$ \\ ${ }^{1}$ Faculty of Environmental Engineering, The University of Kitakyushu, Japan \\ ${ }^{2}$ University of Florence LINV Kitakyushu Research Center, Japan
}

${ }^{3}$ Paris Interdisciplinary Energy Research Institute (PIERI), France

${ }^{4}$ SARAS Co. Ltd, Japan

${ }^{5}$ Royal Cooperation, Japan

*Corresponding author: Tomonori Kawano, Department of Life and Environment Engineering, The University of Kitakyushu, Japan.

Submission: 㘹April 05, 2018; Published: 門 September 12, 2018

\section{Introduction}

Our team at International Photosynthesis Industrialization Research Center (IPIRC), University of Kitakyushu, aims to uncover and develop the systems for sustainable yield of energy such as fuels through science and engineering of both natural and artificial photosynthesis under collaborations with industries and foreign institutions. The natural systems under collaborations include projects with European institutions such as Univ. Paris-Diderot (France) and Univ. of Florence (Italy) for bio-oil production from a Mediterranean halophyte grown on the non-agricultural lands and a collaborative project held in Kitakyushu city, for producing the jet fuel using marine micro-algae run by Electric Power Development Co., Ltd, as we have developed an efficient protocols for extraction of bio-oils from bio-materials [1]. On the other hand, artificial systems under collaborations include a project (with Royal Corp. Ltd. and Sankyu Inc.) for developing a photosynthetic carbon fixation (dark reaction)-mimicking mechanism for absorption and fixation of carbon dioxide $\left(\mathrm{CO}_{2}\right)$ followed by carbon utilization and production of fuels (synthetic diesel).

As the aforementioned Royal Corp. Ltd. has launched, under the auspices of the presidential office of the government of Philippine, a pilot plant in the suburb of Manilla, Philippine, empirically aiming for the process allowing the atmospheric $\mathrm{CO}_{2}$ to be captured in the oil/methanol/water mixture for further direct reaction yielding hydrocarbons under mild conditions in the presence of several types of catalysts; the priority mission for our academic team was to mimic the phenomena in the laboratory bench-top scale based on the working hypothesis possibly explaining the phenomena. Therefore, our team has been intensively engaged in development of novel artificial photosynthetic model reactions which allow reversible uptake and/or release of atmospheric $\mathrm{CO}_{2}$ [2], followed by production and/or consumption of simple organic molecules in laboratory scale in vitro systems. The approach towards such artificial photosynthesis research involves the development and use of organic and inorganic catalysts (artificial enzymes) employed by analogies to redox active natural enzymes or proteins. Here, we briefly present the hypothetical model for enhanced capturing of atmospheric $\mathrm{CO}_{2}$ through finely mixed oil/methanol/water system containing model inorganic catalyst mixture, and the minimal supportive data to date [3].

Working Hypothesis for Carbon Capture and Utilization for Fuel Production based on the Mass-Balance Analysis with the Pilot Plant (Royal Cooperation, Manilla, Philippine)

In Philippine, a model pilot plant originally designed for waterbased refinement (removal of air-pollution precursors) of diesel fuel was installed (Figure 1). Interestingly, several engineers at the Royal Cooperation have noticed that the volume of diesel recovered after aquatic washing process proceed the original volume of the fuel loaded onto the system, suspecting that there would be a phenomenon with unknown mechanism are ongoing. Chemical analyses (Figure 2) performed for comparison of the original diesel and recovered diesel suggested that quality of tow samples is almost identical having identical density and similar elemental compositions. However, gas chromatography suggested that hydrocarbons with slightly elongated chains could be observed in the recovered sample possibly suggesting the possible relationship between the enhanced yield of fuel and elongation of the carbon chain in the hydrocarbons. There would 
be two major reactions during $\mathrm{CO}_{2}$ utilizing production in the pilot plant producing synthetic diesel, namely, [2] initial start-up reaction requiring template hydrocarbons and methanol yielding intermediate hydrocarbons with concomitant elimination of water molecules, and [1] massive reactions lengthening the hydrocarbon chains requiring the template hydrocarbons (and the intermediate hydrocarbons), $\mathrm{CO}_{2}$, and water molecules. In support of above view, preliminary tests showed that oil production was sensitive to blockade of the flow of air and largely enhanced.

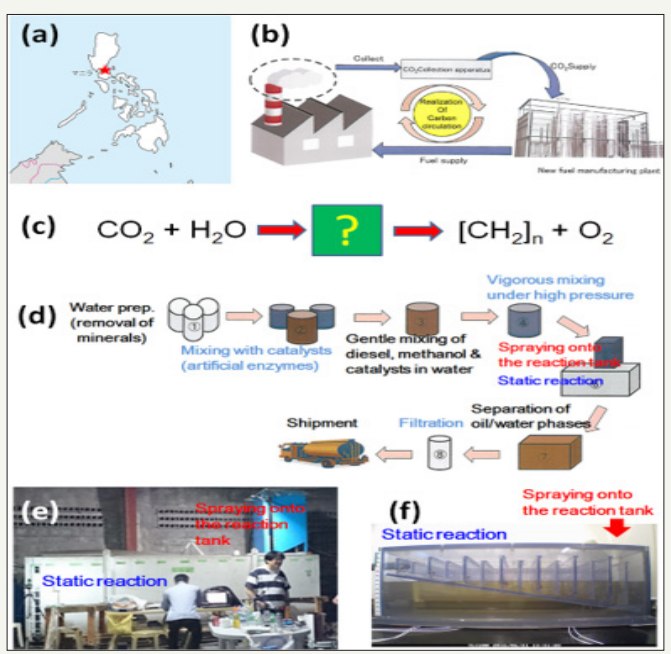

Figure 1: Learning from empirically designed pilot plant run in Philippine.

A. Location of the model pilot plant run for 5 years in Manila, Philippines.

B. Proposed concept of carbon circulation mimicking the photosynthetic fixation.

C. A novel photosynthesis-inspired carbon-fixation model concomitantly releasing oxygen as byproduct.

D. Schematic flows of fuel production processes via conversion (transformation) of atmospheric $\mathrm{CO}_{2}$ in the presence of activated catalysts.

E. Spraying apparatus and reaction tank installed in the pilot plant.

F. Transparent 1/1000-scaled model reaction tank for laboratory tests.

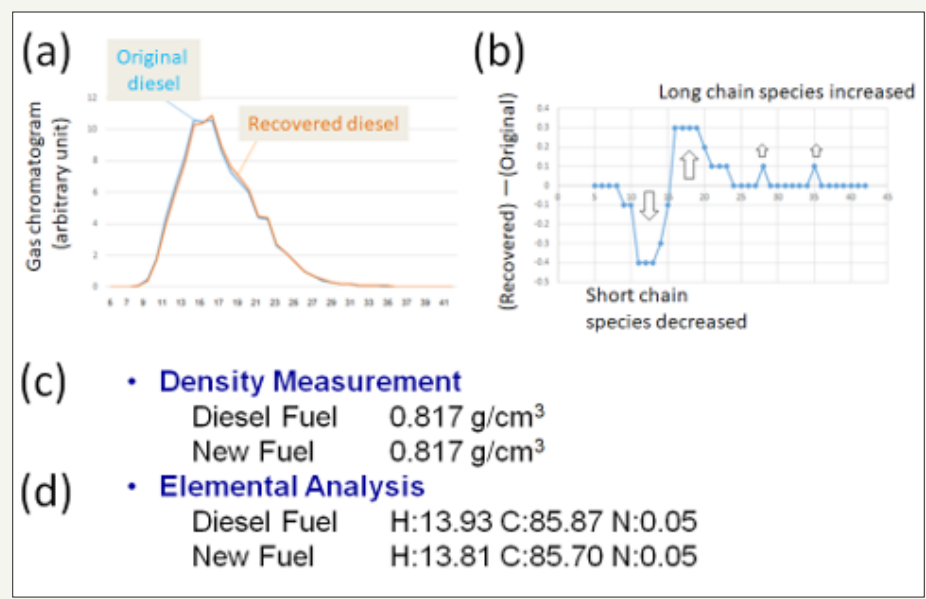

Figure 2: Chemical properties of recovered fuel.
A. Gas chromatograms of original and recovered fuels.
B. Differentially displayed changes in chain length of hydrocarbons.
C. Density of fuels compared.
D. Elemental compositions in original and recovered fuels.

Based on the repeatedly performed mass-balance analyses for the model pilot plants operated in/around Manila, the estimated contribution of the reaction (Figure 3) in which $\mathrm{CO}_{2}$ is the sole carbon source for elongation of hydrocarbon chains (addition of $\mathrm{CH}_{2}$ ), we have estimated that de novo production of 1lt of fuel is equivalent to capturing of $2.81 \mathrm{t}$ of $\mathrm{CO}_{2}$, suggesting that newly 
recovered diesel can be considered as a novel type of fuel converted (transformed) by addition of atmospheric $\mathrm{CO}_{2}$ in the presence of activated catalysts. New diesel fuel can be thus considered "carbon neutral" by analogy to biofuels produced via capturing atmospheric
$\mathrm{CO}_{2}$ through natural photosynthesis, thus, this process could be viewed as a novel artificial photosynthesis corresponding to the dark reactions.

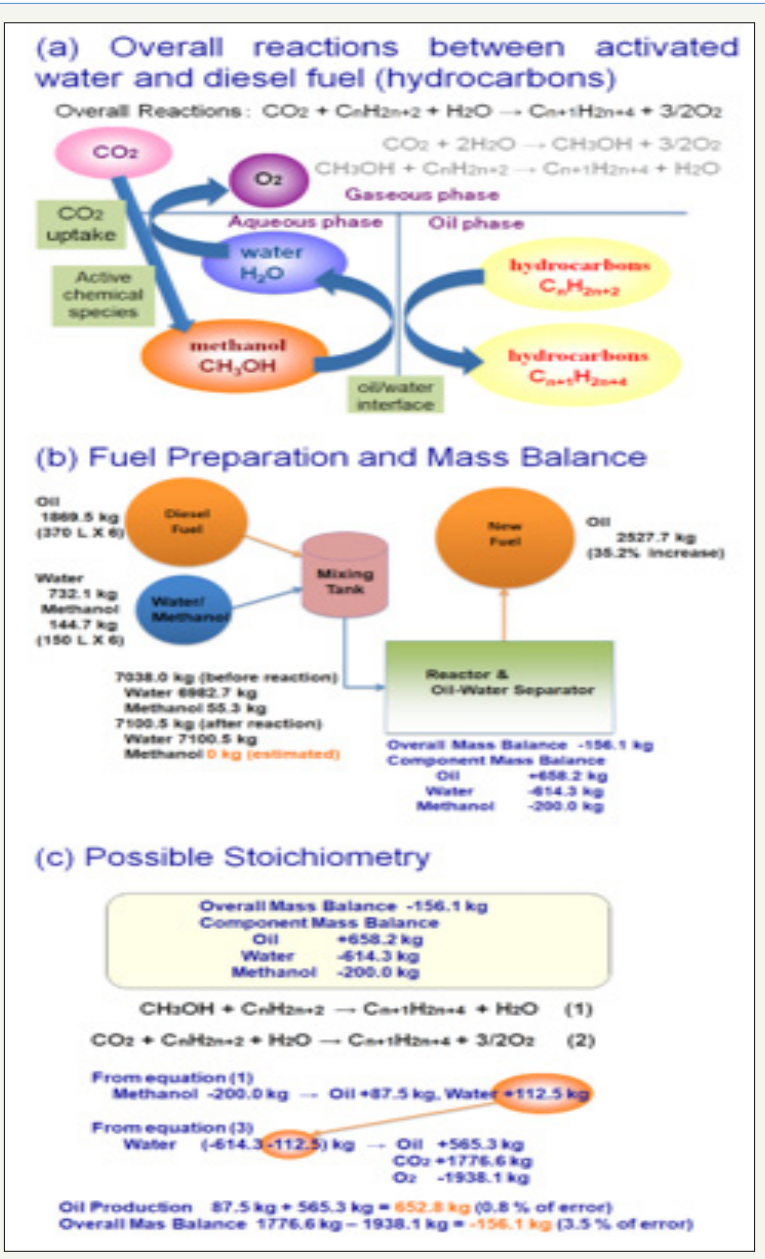

Figure 3: Proposed overall equations and stoichiometry for the catalyzed $\mathrm{CO}_{2}$ uptake and fixation into organic molecules focusing on the roles of methanol, $\mathrm{CO}_{2}$, and original hydrocarbons, as the priming agent, major carbon source, and the template molecules.

A. Overall reactions consisted of two sub-reactions occurring in the interfaces among gaseous, aquatic and oil phases in the finely mixed reaction system.

B. Mass balance of the model system.

C. Possible stoichiometric explanations.

\section{Likely Roles of Redox Reactions Catalyzed by Catalyst} Mixture

As the aqueous media used for refinement of fuels, catalysts (calcium-rich natural zeolite mixture with supplementation of plant enzymes) were suspended, these catalyst mixtures provided by Chubu enzyme Co. Ltd (Aichi, Japan) could be attributed to the phenomena of interest. Notably, water suspended with these calcium-rich zeolite mixtures drastically elevated the level of superoxide anion radical suggesting the continuous production of radical species is catalyzed. Detection of superoxide was performed with chemiluminescence using Cypridina luciferin analog as the superoxide-specific probe according to the protocols used for enzymatic production of superoxide in the aqueous system [3].
The pilot plant empirically designed for diesel fuel refinement was scaled down by $1 / 1000$ (1/10 width, $1 / 10$ height, $1 / 10$ depth) with transparent acryl models (Figure 1), so that ongoing changes in the volume could be visualized. In this model, catalytic water with suspended catalyst mixture (actively producing superoxide, data not shown) was loaded. Following the high-pressure injection of well-mixed oil/methanol/diesel (prepared with the ratio proportional to the original protocol shown in Figure 3, increase in the oil phase could be initiated at the compartment having thin layer of oil low-density emersion mixture and atmospheric phase meet (Figure 4). Interestingly, the level of superoxide showed tendency to be elevated following injection of fuel mixture coinciding the increase of oil. 


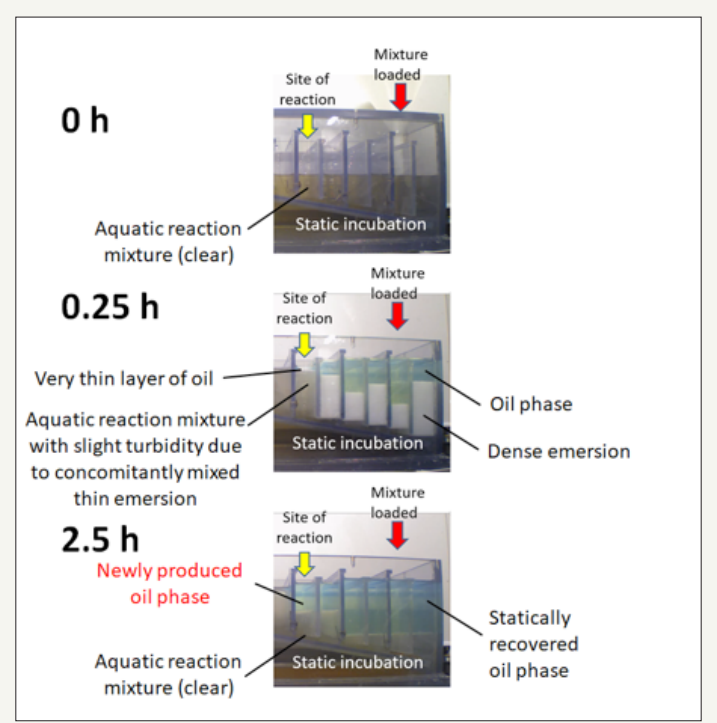

Figure 4: Identification of the site of reaction using 1/1000-scalled transparent model mimicking the phenomenon observed in the pilot plant. Reaction set-up prior to (top), immediately after (middle), and after (bottom) the injection of oil/methanol/catalytic water mixture onto the aquatic reaction mixture containing methanol and catalytic water were continuously monitored.

\section{Simplified Model and Possible Mechanism for Absorbing of Carbon (From the CO2 in the Air) to the Hydrocarbons}

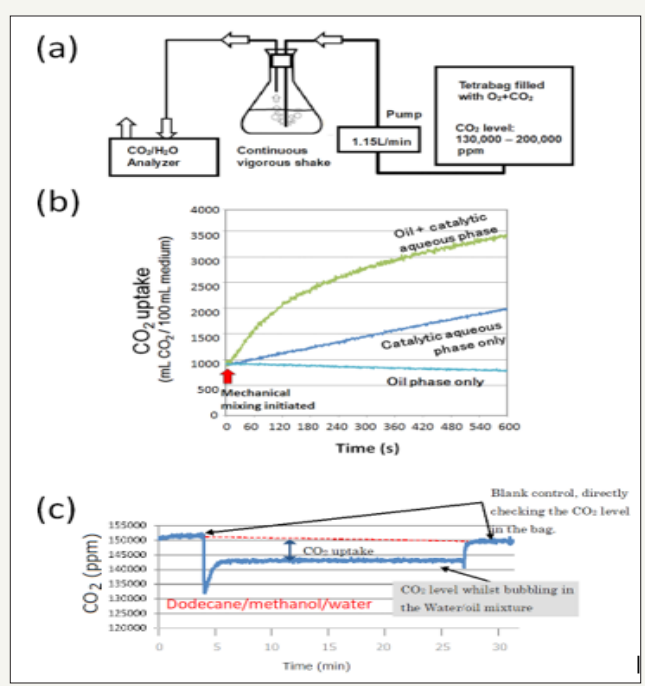

Figure 5: Simplified system for monitoring the involvement of $\mathrm{CO}_{2}$ and water during the catalytic water-assisted synthesis of hydrocarbons
A. Laboratory-scale model experiments for stoichiometric analysis.
B. Synergistic roles of catalytic aqueous mixture and oil for enhanced uptake of $\mathrm{CO}_{2}$ from the air phase.

C. Continuous monitoring of $\mathrm{CO}_{2}$ uptake by dodecane-containing mixture. Composition of reaction mixture: $100 \mathrm{~mL}$ water,

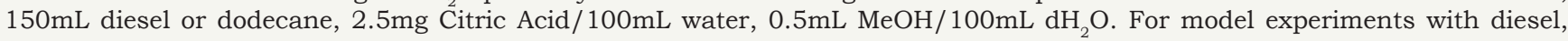
suspension of calcium-rich zeolite (Chubu Enzyme Co. Ltd.) was used as catalyst. For model experiment with dodecane, 20mg human hemoglobin $(\mathrm{Hb}) / 1 \mathrm{~mL} 100 \mathrm{Mm}$ Phosphate buffer $(\mathrm{pH} 7)$ supplemented with $2.7 \mathrm{mg} \mathrm{FeSO}{ }_{4}^{*} 7 \mathrm{H}_{2} \mathrm{O}$ was used as model redox catalysts.

Based on the proposed overall reactions in the pilot plant (Figure 3), we have focused on the utility of $\mathrm{CO}_{2}$ and water as the carbon and hydrogen sources contributing to the formation of hydrocarbons. For assessing the stoichiometry involving $\mathrm{CO}_{2}$ and water, we employed a simplified model which allows continuous monitoring of $\mathrm{CO}_{2}$ and vapor of water flow out by monitoring with $\mathrm{CO}_{2} / \mathrm{H}_{2} \mathrm{O}_{2}$ analyzer (LI-840A, LI-COR, Lincoln, NE, USA) (Figure
5). We have confirmed that uptake of atmospheric $\mathrm{CO}_{2}$ by liquid phases could be maximized when both the catalytic water and the oil (diesel) co-exist in the system (Figure 5). In this model system, containing either of commercial diesel or a model hydrocarbon (dodecane), uptake of $\mathrm{CO}_{2}$ (Figure 5, the case of dodecane shown) and loss of water were continuously traced. We have evaluated the increase of oil phase accompanying the $\mathrm{CO}_{2}$ uptake by an emulsion 
with catalyst-suspended water and oil (commercial diesel or dodecane) as summarized in Table $1 \& 2$. With diesel and calciumrich zeolite, stoichiometry of carbon and water nicely matches the yield of oil while dodecane model experiment employing $\mathrm{Hb}$ showed a similar stoichiometric match with lesser extent.

Table 1: Mass-balance in the Diesel/Methanol/Water system.

\begin{tabular}{|c|c|c|c|c|}
\hline Phase & Initial & Final & Changes & Gas in/out Detected \\
\hline Oil & $121.00 \mathrm{~g}$ & $123.32 \mathrm{~g}$ & $2.32 \mathrm{~g}(1.99 \mathrm{~g}$ as $\mathrm{C})$ & $3.78 \mathrm{~L} \mathrm{CO}_{2}$ taken up $\left(7.42 \mathrm{gas} \mathrm{CO}_{2} ; 2.02 \mathrm{gas} \mathrm{C}^{2}\right.$ \\
\hline Water & $100.37 \mathrm{~g}$ & $89.87 \mathrm{~g}$ & $-10.50 \mathrm{~g}$ & $-6.46 \mathrm{~g}($ as vapor $)$ \\
\hline
\end{tabular}

Table 2: Mass-balance in the Dodecane/Methanol/Water system.

\begin{tabular}{|c|c|c|c|c|}
\hline Phase & Initial & Final & Changes & Gas in/out Detected \\
\hline Oil & $111.33 \mathrm{~g}$ & $113.08 \mathrm{~g}$ & $2.34 \mathrm{~g}(2.01 \mathrm{~g}$ as $\mathrm{C})$ & $5.08 \mathrm{~L} \mathrm{CO}_{2}$ taken up $\left(9.98 \mathrm{gas} \mathrm{CO}_{2} ; 3.17 \mathrm{gas} \mathrm{C}\right)$ \\
\hline Water & $100.15 \mathrm{~g}$ & $90.69 \mathrm{~g}$ & $-9.46 \mathrm{~g}$ & $-12.37 \mathrm{~g}($ as vapor $)$ \\
\hline
\end{tabular}

Likely Mechanisms $\mathrm{CO}_{2}$ Show High Affinity Towards (thin layer of hydrocarbons) since $\mathrm{CO}_{2}$ show high affinity towards Hydrocarbons hydrocarbons [3-5]. Note that this type of phenomena can be also

As shown in the conceptual illustration (Figure 6) representing the movement and conversion of chemical components through the air-oil, oil-water (+methanol), and air-water interfaces within the micro-domains in the water-methanol-oil emersion whipped with air; $\mathrm{CO}_{2}$ in the atmospheric phase enters the phase of oil observed in most organic solvent even in methanol [6-9], thus $\mathrm{CO}_{2}$ can be statically captured (stored) in and readily released out upon mechanical treatment. Taken together, hydrocarbons (including diesel fuel) and organic solvents function as chemical funnel for capturing and concentrating the $\mathrm{CO}_{2}$.

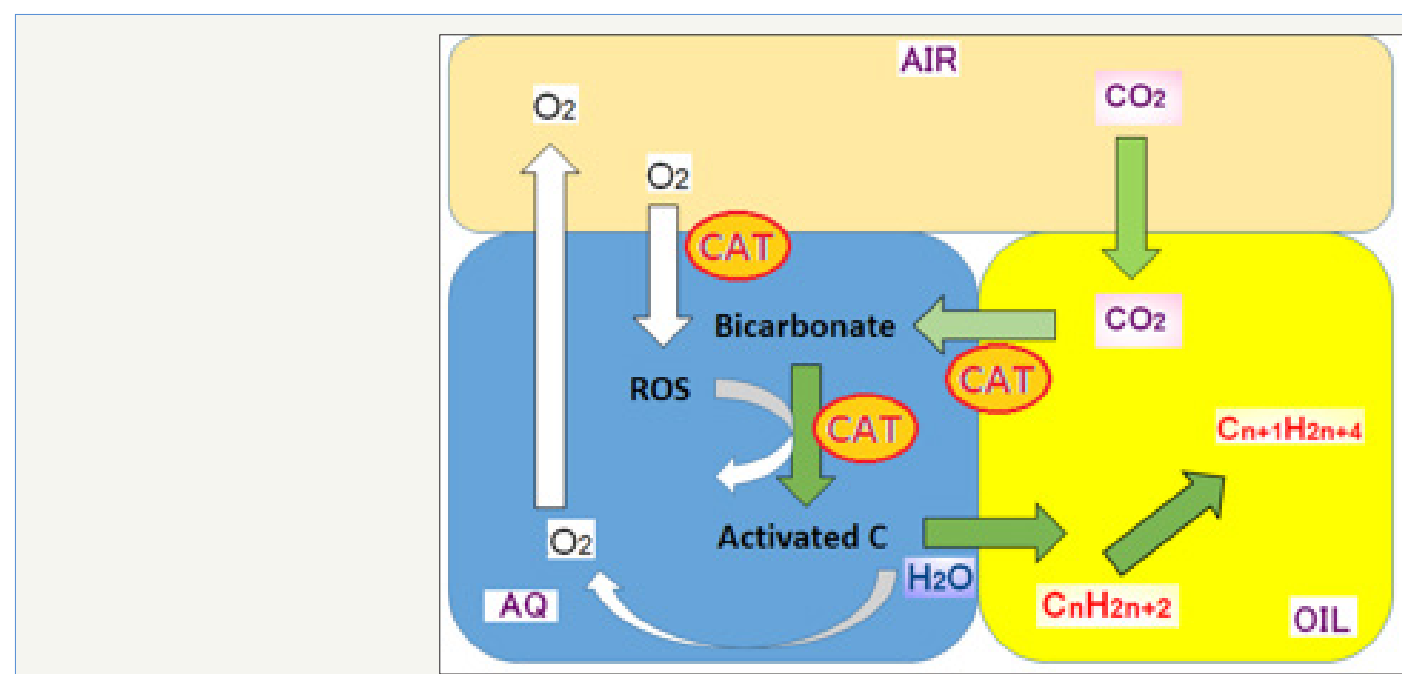

Figure 6: Proposed role of catalysts in the micro-environments consisted of three different phases of materials. CAT: Catalysts; ROS: Reactive Oxygen Species. Illustration provided in the earlier material) presented at international conferences (Kawano T et al. [3] and Uezu k et al. [7,9] University of Kitakyushu International Photosynthesis Industrialization Research Center, 2016).

\section{Enhanced Translocation of $\mathrm{CO}_{2}$}

The capacity for maintaining $\mathrm{CO}_{2}$ in the hydrocarbons must be limited depending on the chemical properties of hydrocarbon chosen and conditions given. In fact, diesel fuel exposed to ambient air is likely saturated and thus no further uptake of $\mathrm{CO}_{2}$ can be expected as shown in the model experiments (Figure 5, oil phase only). Therefore, system must be designed to allow translocation of $\mathrm{CO}_{2}$ from oil phase to aqueous phase in the finely mixed oil and aqueous solution. Again, note that capacity for $\mathrm{CO}_{2}$ in the aqueous phase is limited (readily saturated). In nature, plants were evolved to solve this type of problem, by producing catalysts called carbonic anhydrase which catalyzes the conversion of $\mathrm{CO}_{2}$ into bicarbonate $\left(\mathrm{CO}_{2}+\mathrm{H}_{2} \mathrm{O} \Leftrightarrow \mathrm{H}^{+}+\mathrm{HCO}_{3}^{-}\right)$. One of the roles for catalysts (calcium-rich natural zeolite mixture with supplementation of plant enzymes) employed in our diesel processing system is enhanced conversion of $\mathrm{CO}_{2}$ into bicarbonate in the aqueous phase, thus allowing continuous flow of oil-aqueous $\mathrm{CO}_{2}$ translocation [5-8].

\section{Synergetic Orchestrated Actions of Catalytic Water Phase and Oil Phase}

To prove that radical forming natures (both releasing ROS and C-radicals) in the catalyst-supplemented aqueous phase contribute to enhanced uptake of $\mathrm{CO}_{2}$ by oil phase [9], some laboratory experiments (as one example is mentioned above) were performed and the rate of $\mathrm{CO}_{2}$ uptake was shown to be maximal only when the catalytic water phase and oil phase were finely mixed up. 


\section{Radical Formation as Driving Force for Enhanced Uptake of $\mathrm{CO}_{2}$}

In the aqueous phase, catalysts may play two distinct roles, namely, [2] generation of reactive oxygen species (ROS) such as superoxide anion radical followed by release of hydroxyl radical, through interaction with dissolved molecular oxygen taken up from the air phase, and [1] generation of carbon-derived radical species coupled to the reactions with ROS. The resultant carbon-derived radical species re-enters the oil phase and react with hydrocarbons. These processes might be the driving force required for creating capacity for further (continuous) $\mathrm{CO}_{2}$ uptake in the water and oil phases and for translocation within the emersion complex. At present, model for several putative carbon-derived radical species are under intensive research activity and the relationship between the radical formation and carbon-fixation (materialized as increase in oil) are studied (unpublished results and hypothetic models) [10].

\section{Acknowledgement}

We acknowledge the organizing and scientific committee of the international symposium on Plant Signaling and Behavior 2015 held in Paris for kindly offering the chance to report our idea and key primitive data in front of international audience for the first time dating $29^{\text {th }}$ June 2015 which may crucial for showing the priority of the research. Further confirmations followed as presented (June-July 2017).

\section{References}

1. Nakamura A, Hara Y, Kawano T (2017) Dewatering and extraction of hydrophilic solutes and essential oils from cryo-preserved lemon peels using liquefied dimethyl ether. Solvent Extraction Research and Development, Japan 24(1): 37-45.

2. Comparini D, Uezu K, Kawano T (2015) Monitoring of sonochemically released carbon dioxide from a methanol-water system. Solvent Extraction Research and Development, Japan 22(2): 215-221.

3. Kawano T, Kawano N, Hosoya H, Lapeyrie F (2001) Fungal auxin antagonist hypaphorine competitively inhibits indole-3-acetic aciddependent superoxide generation by horseradish peroxidase. Biochem Biophys Res Commun 288(3): 546-551.

4. Uemura (2013) Analysis of molecular dynamics of $\mathrm{CO}_{2} /$ oil dissolution phenomena in enhanced oil recovery. J Comput Chem Jpn 12(4): 222 229.

5. Hara Y, Kikuchi A, Noriyasu A, Furukawa H, Takaichi H, et al. (2016) Batch extraction of oil from rice bran with liquefied low temperature dimethyl ether. Solvent Extraction Research and Development, Japan 23(1): 87-99.

6. Kawano $T$ (2015) Introduction to natural, artificial, and hybrid photosynthesis researches. Plant Signaling and Behavior, Paris, France.

7. Uezu K, Kawano T, Comparini D, Uchimura O (2015) Chemical engineering approach for artificial photosynthesis system. PSB2015: Plant Signaling and Behavior, Paris, France.

8. University of Kitakyushu International Photosynthesis Industrialization Research Center (2016) Artificial fuel production system mimicking plant photosynthesis: Poster presentation at G7 Kitakyushu energy ministerial meeting.

9. Uezu K, Kawano T, Uchimura O (2017) Prediction of the reaction scheme for novel artificial photosynthesis system by the chemical engineering approach. $5^{\text {th }}$ International Symposium on Plant Signaling and Behavior Matsue, Japan.

10. Kawano T, Comparini D, Uchimura O, Uezu K (2017) Natural and artificial photosynthesis models for capturing carbon dioxide into fuels. $5^{\text {th }}$ International Symposium on Plant Signaling and Behavior Matsue, Japan.
Creative Commons Attribution 4.0

International License

For possible submissions Click Here

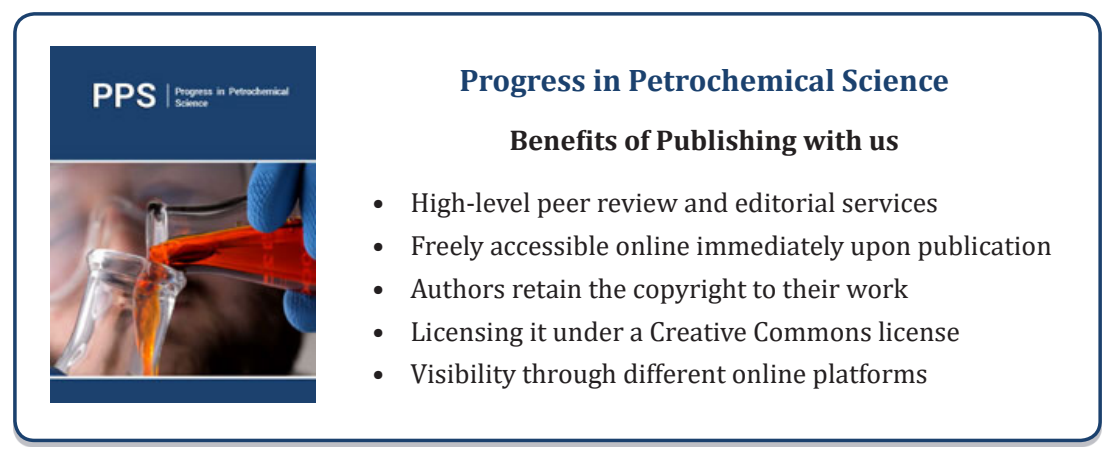

